\title{
The Assessment of the Clinical Effect of the Drug Compatibility and Course of Treatment to the Brucellar Spondylitis
}

\author{
Xin-Ming Yang ${ }^{*}$, Wei Shi ${ }^{2}$, Xian-Yong Meng ${ }^{1}$, Ying Zhang ${ }^{1}$, Ya-Kun Du ${ }^{3}$, Lei Zhang ${ }^{4}$, Yao-Yi Wang ${ }^{1}$ \\ ${ }^{1}$ Department of Orthopaedics, The First Affiliated Hospital of Hebei North University, Zhangjiakou, China \\ ${ }^{2}$ Department of Paediatrics, The First Affiliated Hospital of Hebei North University, Zhangjiakou, China \\ ${ }^{3}$ Department of Neurology, The Children's Hospital of Hebei Province, Shijiazhuang, China \\ ${ }^{4}$ Department of Neurosurgery, The Fourth Affiliated Hospital of Hebei Medical University, Shijiazhuang, China \\ Email: ${ }^{*}$ xxm1120@sohu.com.
}

Received September 6, 2012; revised October 9, 2012; accepted October 19, 2012

\begin{abstract}
Objective: To evaluate five drug treatment regimens in the treatment of Brucella spondylitis. Methods: Patients with clinical symptoms compatible and diagnostic test consistent with Brucella spondylitis were randomly assigned to five drug treatment regimens. Results: Combination therapy with doxycycline, rifampin and sulfamethoxazole for 56 consecutive days showed the highest cure rate of $20 \%$ after a single course and of $85 \%$ after a double course with affectivity rates of 55\% and 95\%. Cure rate and affectivity rate was significant better $(\mathrm{P}<0.05)$ than for patients receiving doxycycline, rifampin and streptomycin for the same period and regimens containing doxycycline were significant better than regimens without this drug. Conclusion: Combination therapy of doxycycline, rifampin and sulfamethoxazole for 8 weeks using one or two full courses should be recommended for Brucella spondylitis.
\end{abstract}

Keywords: Brucellosis; Spondylitis; Clinic Effect; Course of Treatment; Drug Compatibility

\section{Introduction}

Estimating Brucellosis is a infectious allergic diseases caused by bacterium burgeri, which can happened in both human beings and animals, with very broad endemic regions. In recent years, the strain, susceptible population and route of transmission have changed that the bovine type, caprine type and swine type bacterium burgeri can be spread to human beings not only by contacting damaged skin or membrana mucosa directly but also by intaking contaminated food. The global incidence is increasing, especially the city incidence. Brucellosis has become a disease caused by food from an occupational disease. Bones and joints often invaded by the disease, and the two consecutive segments of the spine are most commonly involved, which can triggered spondylitis and discitis leading to large devastating spine combined with spinal instability and spinal cord, cauda eguina or root compression [1-8] (Figure 1). Related clinical application finds that the classic drug treatment and compatibility have less clinical effective to the Brucella spondylitis disease and easily to relapse [9-14]. So, searching for sensitive drugs, adjusting and optimizing the compatibil

${ }^{*}$ Corresponding author. ity of the program and getting the best method of treatment have a positive effect to cure early Brucella spondylitis, reduce disability, avoid the surgical risk and prevent the recurrence which are reported as follows:

\section{Methods}

\subsection{Design}

The design of the topic is study of screeningclinical sensitive drugs, compatibility programs and courses to cure Brucella spondylitis.

\subsection{Time and Study Site}

The study was approved and granted by the Ethics Committee of the First Affiliated Hospital of Hebei North University and completed in the First Affiliated Hospital of Hebei North University in Zhangjiakou City, Hebei Province, China, between January of 2006 and July of 2011.

\subsection{Adoption Standard}

The epidemiological history of the patients (including the living area, gender, age, occupation, disease location, 

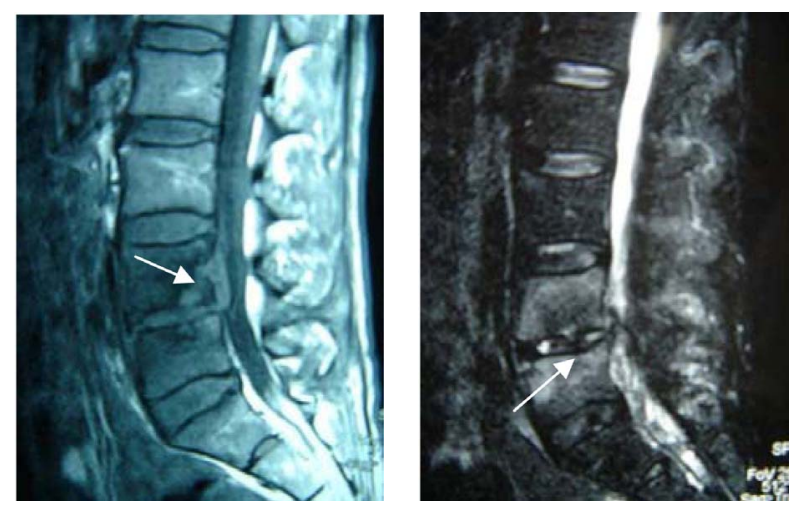

Figure 1. MRI before operation arrow indicated that the sclerotin of the vertebrae and the Intervertebral disc were destructed, there was prevertebral, intervertebral, and epidural abscess, and the cauda eguina was compressed on the corresponding level.

number of diseased vertebral body, focal distribution), clinical manifestations, imaging features (X-ray, CT, MR) and the clinical specific serological examination such as Serum tube agglutination (SAT), rose bengal plate agglutination test (RBP), enzyme-linked immunosorbent assay (ELISA) and so on, which were consistent with the diagnostic criteria of brucellosis published by Endemic Disease Prevention and Cure Bureau of Ministry of Public Health $[11,12,15]$.

\subsection{Elimination Standard}

The skeleton wasn't mature, immunosuppressive drugs were used, the patients had other spinal infection before or at present, the spine was unstable, there was abscess around the vertebra, severely damaged body of vertebra, epidural abscess in the vertebral canal, granuloma or spinal cord, cauda eguina or root compression, which were surgical indications (Figures 1-3).

\subsection{Subjects}

All of the 200 cases were consistent with the adoption standard, coming from Zhangjiakou of Hebei Province and six counties of Neimenggu in China, of which 55 cases from pastoral area (56.12\%) and 43 cases from non-pastoral areas (43.87\%). Male 91 cases, female 109 cases, and the ratio of male to female is 1:1.19. Age Distribution: Maximum age is 60 years old, minimum age is 21 years old, 25 cases are 21 - 30 years old, 48 cases are 31 - 40 years old, 92 cases are 41 - 50 years old , 35 cases are 51 - 60 years old, average age in this group is $45 \pm 3.5$ years old. Diseased parts: There are 172 cases with two vertebral bodies involved, of which 4 cases with T10-11, 4 cases with T11-12, 6 cases with T12L1, 6 case with L1-2, 20 cases with L2-3, 80 cases with L3-4, 52 case with L4-5.There are 28 cases with three vertebral bodies involved, of which 3 cases with T8-10, 5 cases

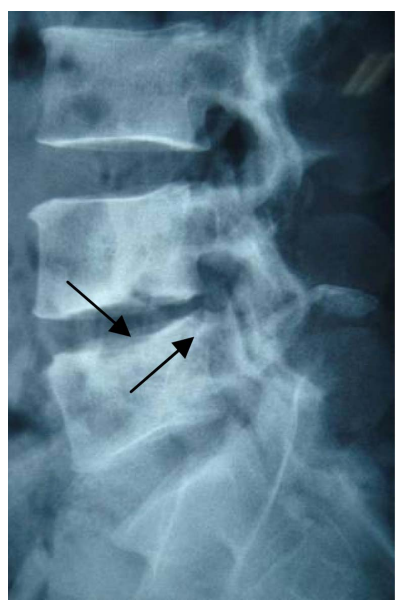

Figure 2. The lateral $\mathrm{X}$-ray film before operation arrow indicated that the intervertebral space was destructed and became narrow, the sclerotin of the vertebrae had multiple destructive focuses and bone sclerosis.

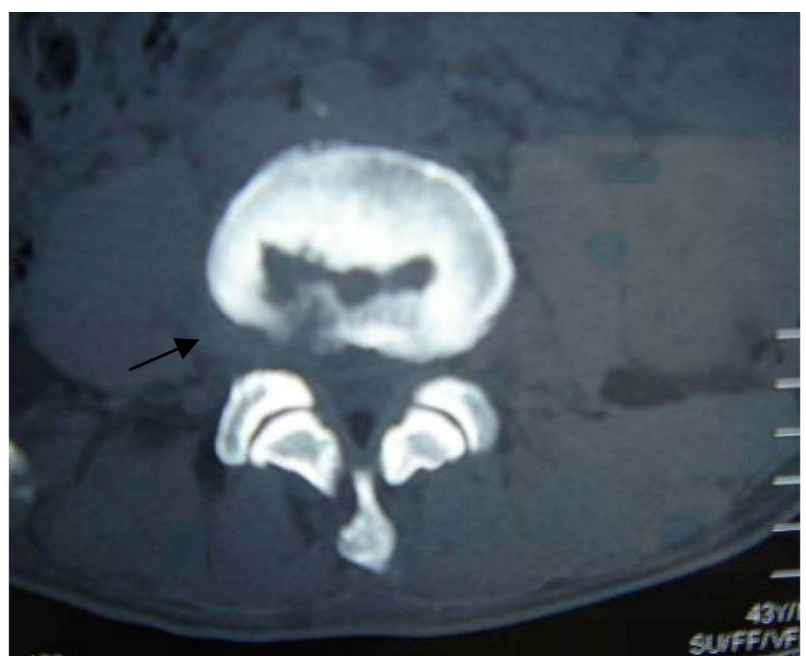

Figure 3. CT before operation arrow indicated there were multiple destructive focuses in the vertebrae which involved the posterior border of the vertebrae, the periphery of the focus and the articular process became sclerified, the intervertebral disc was destructed and there was small abscess around the vertebrae.

with L2-4, 13 cases with L3-5, 7 cases with L4-5S1. Focus of infection distribution: lumbar vertebrae were more than thoracic vertebrae, involving L4 with the highest incidence rate (157/200 cases) 78.5\%, L3 with 59\% and L5 with $36 \%$. All of the 200 patients in this group had a past or present history of intermittent low-grade fever, the body temperature of whom is not more than $38.5^{\circ} \mathrm{C}$, accompanied by fatigue, night sweats, back pain, muscle spasm and constrained movements of spine. 200 cases were divided into five groups by a doctor according to pastoral area and non-pastoral area blindly (Table 1). The subjects without drug hypersensitivity history and contraindication were informed before treatment about 
the drug names, the content of drug combination program and the related treatment method, and obtain their informed consent. The general data of the patients in the five groups had comparability and the difference had no significance ( $\mathrm{P}>0.05$, Table 1).

\subsection{The Standard to Assess the Curative Effect $[11,12]$}

1) Healing well. The temperature recovered to normal, the clinical symptoms and physical signs disappeared and the physical strength and labor force recovered. MRI showed that the abscess of the intervertebral space or the vertebral body disappeared or became calcified and the circumscription of the initial focus of infection became clear. RBPT was negative; 2) Improved. The temperature recovered to normal, the clinical symptoms and physical signs disappeared and the physical strength and labor force recovered in principle. MRI showed that the abscess of the intervertebral space or the vertebral body was absorbed partly and the circumscription of the initial focus of infection became less clear and the damaged sclerotin had the sign of recovery. RBPT was negative; 3 ) Ineffective. The temperature fluctuated, the clinical symptoms, physical signs and MRI had no significant change or improvement, RBPT was negative or positive, or brucellar spondylitis recurred. The healing rate (quantity of healing well/quantity of the group) and the effective rate (quantity of healing well and improved/quantity of the group) were used as the evaluation index.

\subsection{Therapeutic Method}

All of the patients were protected by corset during the drug treatment and rested in bed for more than 3 weeks in every course of treatment. Bacterial culture and susceptibility test were positive in only 43 cases and aspiration-needle biopsy was performed in the other 157 cases. The patients in the five groups were treated with classic drugs for brucellar spondylitis. Five sensitive drugs and five compatibility programs were chosen according to the result of the bacterial culture and susceptibility test of the
43 cases. The same dose of a drug, medication and time were adopted to each identical group that Doxycycline $0.1 \mathrm{~g}$, bid, consecutively for 56 days and the double dose for the first time; Rifampicin $0.6 \mathrm{~g}$, qd, consecutively for 56 days; Sulfamethoxazole $1.0 \mathrm{~g}$, bid, consecutively for 56 days; Levofloxacin $0.5 \mathrm{~g}$, qd, consecutively for 56 days; Streptomycin $0.75 \mathrm{~g}$, intramuscular injection, qd, for 21 days. In Group 1 (40 cases) Doxycycline, Rifampicin and Sulfamethoxazole were used; Doxycycline, Rifampicin and Levofloxacin for group 2 (40 cases); Doxycycline, Rifampicin and Streptomycin for group 3 (40 cases); Doxycycline, Sulfamethoxazole and Streptomycin for group 4 (40 cases); Rifampicin, Streptomycin and Sulfamethoxazole for group 5 (40 cases). One course of treatment was 56 days. After two courses of treatment, the third course of treatment wouldn't be performed if the effective rate was lower than $60 \%$ reported by literature but the programme with the highest cure rate and effective rate would be adopted again. There was an interval of 7 days between every two courses of treatment and hepatic and renal functions were examined during the interval.

\subsection{Analytical Method of the Data}

The data of this study were analyzed by SPSS 15.0 statistical packages. The measurement data were expressed in the form of mean \pm standard deviation and one- factor analysis of variance was adopted to make the comparison among groups. Chi-square test was used to analyze numeration data. The size of test was 0.05 .

\section{Results}

\subsection{The Laboratory Examination and Pathological Examination Result before Drug the Rapy}

The PCR sensitivity analysis of the bovine type, caprine type and swine type bacterium burgeri was performed to all of the 200 cases, of which 155 cases were positive and the positive rate was $77.5 \%$, caprine type 80 cases, bovine type 51 cases and bovine type 24 cases. The susceptibility

Table 1. Clinical data of brucellar spondylitis of five groups.

\begin{tabular}{|c|c|c|c|c|c|c|c|}
\hline Group & Case & Pastoral area & Non-pastoral area & Male/female & Mean age (year) & $\begin{array}{l}\text { Two vertebral } \\
\text { bodies involved }\end{array}$ & $\begin{array}{l}\text { Three vertebral } \\
\text { bodies involved }\end{array}$ \\
\hline Group 1 & 40 & 21 & 19 & $19 / 21$ & $46 \pm 3.7(21-60)$ & 34 & 6 \\
\hline Group 2 & 40 & 18 & 22 & $19 / 21$ & $46 \pm 3.1(22-58)$ & 33 & 7 \\
\hline Group 3 & 40 & 20 & 20 & $18 / 22$ & $45 \pm 3.4(24-57)$ & 35 & 5 \\
\hline Group 4 & 40 & 22 & 18 & $18 / 22$ & $44 \pm 3.3(25-54)$ & 35 & 5 \\
\hline Group 5 & 40 & 20 & 20 & $17 / 23$ & $45 \pm 4.3(22-60)$ & 35 & 5 \\
\hline Result & - & \multicolumn{2}{|c|}{$\begin{array}{l}\chi^{2}=0.88 \\
P=0.975\end{array}$} & $\begin{array}{l}\chi^{2}=0.28 \\
P=0.991\end{array}$ & $\begin{array}{l}F=2.18 \\
P=0.07\end{array}$ & \multicolumn{2}{|c|}{$\begin{array}{l}\chi^{2}=0.66 \\
P=0.956\end{array}$} \\
\hline
\end{tabular}


test was positive in only 43 cases. Bacterium burgeri was sensitive to Doxycycline, Rifampicin, Sulfamethoxazole, Levofloxacin and Streptomycin, medium sensitive to Penicilin, Cidomycin and Clindamycin and resistant to Isonicotinyl hydrazide and Ethambutol. The other 157 cases were diagnosed as brucellosis by aspiration-needle biopsy. Histiocyte proliferation in the diseased region, proliferating nodus and granuloma, a lot of monocytes, leucocytes, granulocytes and eosinocyte invasion and nodosity focus of infection made of epithelioid cells can be seen by microscope (Figure 4).

\subsection{Comparison of Clinical Effect on Different Course of Treatment}

The improved and infectious patients in all the five drug compatibility groups after one course of treatment were given the second course of treatment and the third course of treatment was carried out by means of this kind. Comparing the cure rate and effective rate of the second course of treatment with those of the first course of treatment in the first four groups, the difference had statistical significance ( $\mathrm{P}<0.05$, Tables 2(a)-(d)) while comparing the cure rate and effective rate of the second course of treatment with those of the first course of treatment in Group 5,the difference had no statistical significance ( $\mathrm{P}>0.05$, Tables 2(a)-(e)).Comparing the cure rate and effective rate of the third course of treatment with those of the second in the first, second and third group the difference had no significance $(\mathrm{P}>0.05$, Tables 2(a)-(d)). Because the effective rate of the second course of treatment in Groups 4 and 5 was lower than $60 \%$ reported by literature [1], the programme was stopped and the programme of the first group was used as the second course of treatment to treat the infectious and im-

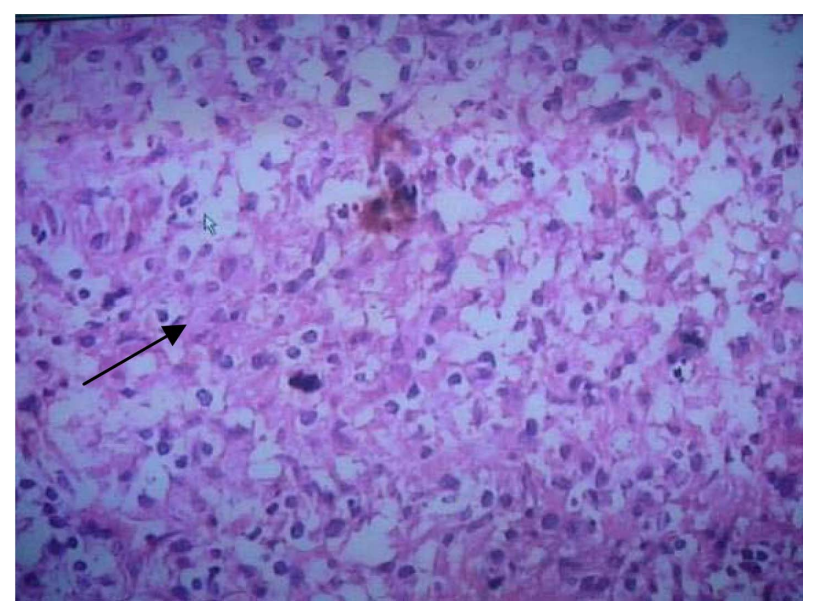

Figure 4. Histopathology examination during operation (HE $\times 100)$ arrow indicated that there were tissular and cellular hyperplasia, proliferating nodule, and granuloma in the focus. proved patients. By comparing the cure rate and effective rate of the second course of treatment with those of the first course of treatment, the difference had statistical significance $(\mathrm{P}<0.05$, Table 2(f)).

\subsection{Comparison of Clinical Effect between the Groups with Doxycycline and Those without It}

The first, second, third and fourth group with Doxycycline on the second course of treatment were made up into one unit and the fifth group without Doxycycline on the second course of treatment was made into another unit. The cure rate and effective rate of the two units were compared and the difference had statistical significance $(\mathrm{P}<0.05$, Table 3$)$.

\subsection{Evaluate the Effect of Single Drug by Comparing the Clinical Effect among Groups}

The clinical effect of the five drug compatibility groups after two courses of treatment (Table 4). Comparing the clinical effects of different drug compatibility among groups, the difference among the other groups had statistical significance except the difference between Groups 2 and 3 ( $\mathrm{P}<0.05$, Table 5).

\subsection{The Hepatic and Renal Function during the Interval of Course of Treatment}

The hepatic and renal function of all the 200 cases was normal after the first and second course of treatment. Eighteen patients had abnormal hepatic and renal function in 120 cases after the third course of treatment, of which there were 5 cases in Group 1, 7 cases in Group 2 and 6 cases in Group 3. All the patients with abnormal hepatic and renal function stopped the drugs and the hepatic and renal function recover to normal after the 14 to 35 day hepatic and renal prevention therapy.

\section{Discussion}

Brucellar spondylitis is treated mainly by drugs. Choosing the correct drugs and course of treatment is benefit to the cure of diseased region, relief of the pain and decrease of complications [9-12,15-18]. The principle instituted by the six alliance bulletin of WHO is using Tetracycline and Streptomycin or using the same kind of drugs as alternative medicine. The fairly ideal programme is that taking Deoxycycline $0.1 \mathrm{~g}$, qd orally consecutively for 45 days and the double dose for the first time and using Streptomycin $0.75 \mathrm{~g}$ intramuscular injection, qd, for 14 days or using Gentamycin 160,000 U intramuscular injection, bid, for 7 days as the alternative medicine of Streptomycin. Many domestic and overseas scholars find that this method is not very effective, the 
Table 2. (a) Comparison of clinical effect on different course of treatment of Group 1; (b) Comparison of clinical effect on different course of treatment of Group 2; (c) Comparison of clinical effect on different course of treatment of Group 3; (d) Comparison of clinical effect on different course of treatment of Group 4; (e) Comparison of clinical effect on different course of treatment of Group 5; (f) Comparison of clinical effect on different course of treatment of the incurred cases in Group 4 and 5 after using the programme of Group 1.

(a)

\begin{tabular}{|c|c|c|c|c|c|c|c|c|c|c|}
\hline Course of treatment & Case & Healing well & Improved & Infectious & Cure rate (\%) & $\chi^{2}$ & $\mathrm{P}$ & Effective rate (\%) & $\chi^{2}$ & $\mathrm{P}$ \\
\hline One course of treatment & 40 & 8 & 14 & 18 & 20 & 33.89 & $<0.001$ & 55 & 17.07 & $<0.001$ \\
\hline Two courses of treatment & 40 & 34 & 4 & 2 & 85 & 0 & 1 & 95 & 0 & 1 \\
\hline Three courses of treatment & 40 & 34 & 5 & 1 & 85 & - & - & 97.5 & - & - \\
\hline
\end{tabular}

Note: effective rate — quantity of healing well and improved/quantity of the group.

(b)

\begin{tabular}{|c|c|c|c|c|c|c|c|c|c|c|}
\hline Course of treatment & Case & Healing well & Improved & Infectious & Cure rate (\%) & $\chi^{2}$ & $\mathrm{P}$ & Effective rate (\%) & $\chi^{2}$ & $P$ \\
\hline One course of treatment & 40 & 6 & 10 & 24 & 15 & 20.83 & $<0.001$ & 40 & 10.03 & 0.002 \\
\hline Two courses of treatment & 40 & 26 & 4 & 10 & 65 & 0 & 1 & 75 & 0.07 & 0.793 \\
\hline Three courses of treatment & 40 & 26 & 5 & 9 & 65 & - & - & 77.5 & - & - \\
\hline
\end{tabular}

(c)

\begin{tabular}{|c|c|c|c|c|c|c|c|c|c|c|}
\hline Course of treatment & Case & Healing well & Improved & Infectious & Cure rate (\%) & $\chi^{2}$ & $\mathrm{P}$ & Effective Rate (\%) & $\chi^{2}$ & $P$ \\
\hline One course of treatment & 40 & 6 & 8 & 26 & 15 & 17.28 & $<0.001$ & 35 & 8.46 & 0.004 \\
\hline Two courses of treatment & 40 & 24 & 4 & 12 & 60 & 0 & 1 & 70 & 0 & 1 \\
\hline Three courses of treatment & 40 & 24 & 4 & 12 & 60 & - & - & 70 & - & - \\
\hline
\end{tabular}

(d)

\begin{tabular}{ccccccc}
\hline Course of treatment & Case & Healing well & Improved & Infectious & Cure rate (\%) & Effective rate (\%) \\
\hline One course of treatment & 40 & 4 & 4 & 32 & 10 & 20 \\
Two courses of treatment & 40 & 15 & 3 & -22 & 37.5 & 45 \\
$\chi^{2}$ & - & - & - & - & 0.35 & 5.70 \\
P & - & - & - & 0.004 & 0.017 \\
\hline
\end{tabular}

(e)

\begin{tabular}{ccccccc}
\hline Course of treatment & Case & Healing well & Improved & Infectious & Cure rate (\%) & Effective rate (\%) \\
\hline One course of treatment & 40 & 1 & 7 & 32 & 2.5 & 20 \\
Two courses of treatment & 40 & 5 & 4 & 31 & 12.5 & 22.5 \\
$\chi^{2}$ & - & - & - & - & 1.62 & 0.08 \\
P & - & - & - & 0.203 & 0.785 \\
\hline
\end{tabular}

(f)

\begin{tabular}{|c|c|c|c|c|c|c|}
\hline Course of treatment & Case & Healing well & Improved & Infectious & Cure rate (\%) & Effective rate (\%) \\
\hline One course of treatment & 60 & 14 & 19 & 27 & 23.33 & 55 \\
\hline Two courses of treatment & 60 & 52 & 4 & 4 & 86.66 & 93.33 \\
\hline$\chi^{2}$ & - & - & - & - & 48.62 & 23.01 \\
\hline $\mathrm{P}$ & - & - & - & - & $<0.001$ & $<0.001$ \\
\hline
\end{tabular}


Table 3. Comparison of clinical effect between the groups with Doxycycline and those without it.

\begin{tabular}{ccccccc}
\hline Group & Case & Healing well & Improved & Infectious & Cure rate (\%) & Effective rate (\%) \\
\hline With Doxycycline & 160 & 99 & 15 & 46 & 61.87 & 71.25 \\
Without Doxycycline & 40 & 5 & 4 & 31 & 12.5 & 22.5 \\
$\chi^{2}$ & & & & 31.26 & 32.12 \\
$\mathrm{P}$ & & & & $<0.001$ & $<0.001$ \\
\hline
\end{tabular}

Table 4. The clinical effect of different drug compatibility.

\begin{tabular}{ccccccc}
\hline Group & Case & Healing well & Improved & Infectious & Cure rate (\%) & Effective rate (\%) \\
\hline Group 1 & 40 & 34 & 4 & 2 & 85 & 95 \\
Group 2 & 40 & 26 & 4 & 10 & 65 & 75 \\
Group 3 & 40 & 24 & 4 & 12 & 60 & 70 \\
Group 4 & 40 & 15 & 3 & 22 & 37.5 & 45 \\
Group 5 & 40 & 5 & 4 & 31 & 12.5 & 22.5 \\
\hline
\end{tabular}

Table 5. Comparison of clinical effect of different drug compatibility between every two groups.

\begin{tabular}{ccccc}
\hline \multirow{2}{*}{$\begin{array}{c}\text { gomparing } \\
\text { groups }\end{array}$} & \multicolumn{2}{c}{ Cure rate } & \multicolumn{2}{c}{ Effective rate } \\
\cline { 2 - 5 }$\chi^{2}$ & $\mathrm{P}$ & $\chi^{2}$ & $\mathrm{P}$ \\
\hline Group 1 and 2 & 4.27 & 0.039 & 6.28 & 0.012 \\
Group 2 and 3 & 0.21 & 0.644 & 0.55 & 0.459 \\
Group 3 and 4 & 4.05 & 0.044 & 4.11 & 0.043 \\
Group 4 and 5 & 6.67 & 0.01 & 4.53 & 0.033 \\
\hline
\end{tabular}

effective rate is only $60 \%$ and has high recurrence rate $[1,17,19,20]$. Thus, it is necessary to find drugs sensitive to brucellar spondylitis and the drug compatibility and to reevaluate the course of treatment on the base of present drugs.

In this article, 200 cases consistent with the adoption standard were treated for two to three courses of treatment and studied contrastively by choosing five different drugs and making five different drug compatibility programs. The first, second, third and fourth group included Doxycycline and the fifth group didn't include Doxycycline. The general data of the patients in the five groups had comparability and the difference had no significance (P > 0.05, Table 1). Table 2 showed that the patients that didn't cure after one course of treatment should be treated for another course of treatment, if still didn't cure, should be treated for the third course of treatment. The cure rate and effective rate of the second course of treatment was better than those of the first course of treatment and the difference had statistical significance $(\mathrm{P}<0.05)$ by comparing the cure rate and effective rate of the second course of treatment with those of the first course of treatment in the first four groups. By comparing the cure rate and effective rate of the third course of treatment with those of the second course of treatment in Groups
1-3, the difference had no statistical significance $(\mathrm{P}>$ 0.05), which indicated that increasing the time of therapy didn't have obvious effect to brucellar spondylitis but increase the incidence of side effect of the drugs. In this article, after three courses of treatment, 18 patients with abnormal hepatic and renal function stopped the drugs and the hepatic and renal function recover to normal after hepatic and renal prevention therapy, which indicated that increasing the time of therapy is increasing the dose of drugs and the incidence of side effect of the drugs. Thus, choosing two courses of treatment is a fairly appropriate scheme. Table $\mathbf{3}$ showed that the difference between the unit with Doxycycline and the unit without Doxycycline had statistical significance $(P<0.05)$, which indicated that the drug compatibility with Doxycycline had obvious effect to brucellar spondylitis and had fairly high cure rate and effective rate and Doxycycline was the main drug. Table 4 showed that after two courses of treatment, comparing the clinical effect among the five groups, the difference had statistical significance $(\mathrm{P}<0.05)$ and the cure rate and effective rate of the first group was better than those of the other four groups. Table 5 showed that by comparing the clinical effect between every two groups-Group 1 and Group 2, Group 3 and Group 4, and Group 4 and Group 5, the difference had statistical significance $(\mathrm{P}<0.05)$. The effect of the first group was better than that of the second group, except the same drugs Doxycycline and Rifampicin, indicating that the effect of Sulfamethoxine to brucellar spondylitis was better than Levofloxacin. By analogy like this, the effect of Rifampicin was better than Sulfamethoxine by comparing Group 3 and Group 4 and the effect of Doxycycline was better than Rifampicin by comparing Group 4 and Group 5 but the difference between Group 2 and Group 3 had no statistical significance $(\mathrm{P}>0.05)$, indicating there was no difference of 
the effect between Levofloxacin and Streptomycin. Thus, the sequence of the single drug was inferred as follows: Doxycycline $>$ Rifampicin $>$ Sulfamethoxine $>$ Levofloxacin or Streptomycin. Solera $[20,21]$ considered there will not be excellent prospective efficacy no matter using what kind of single drug to treat brucellar spondylitis only drug compatibiligy has the most obvious effect. As the above mentioned, the drug compatibiligy of the first group was the most appropriate, had the highest cure rate and effective rate, had the most obvious effect and there was no side effect of drug compatibility (Figures $\mathbf{5}$ and 6). Performing the scheme of Group 1 to the uncured (infectious and improved ) cases in Group 4 and Group 5 also indicated that the cure rate and effective rate of the second course of treatment were better than those of the first course of treatment and the difference had statistical significance by comparing with the first course of treatment $(\mathrm{P}<0.05$, Table 2(f)). The bacterial culture and susceptibility test in 43 cases showed that bacterium burgeri was sensitive to Doxycycline, Rifampicin, Sul- famethoxazole, Levofloxacin and Streptomycin, medium sensitive to Penicilin, Cidomyci and Clindamycin and resistant to Isonicotinyl hydrazide and Ethambutol.

As the above mentioned, the fairly ideal scheme at present to treat brucellar spondylitis was as follows: 1 chief drugs: Doxycycline $0.1 \mathrm{~g}$, bid, consecutively for 56 days and the double dose for the first time; Rifampicin $0.6 \mathrm{~g}$, qd, consecutively for 56 days; Sulfamethoxazole $1.0 \mathrm{~g}$, bid, consecutively for 56 days. The program of Doxycycline, Rifampicin and Sulfamethoxazole is the first choice, consistent with the chief drugs in Antimicrobial Therapy Manual of Sanford [22,23]. 2 minor drugs: Levofloxacin $0.5 \mathrm{~g}$, qd, consecutively for 56 days; Streptomycin $0.75 \mathrm{~g}$, intramuscular injection, qd, for 21 days. The program of Doxycycline, Rifampicin and Levofloxacin or Doxycycline, Rifampicin and Streptomycin was the second choice. The patients should be treated for two courses of treatment usually with 7 day interval between every two courses of treatment until RBP becomes negative, using the drugs for another two weeks

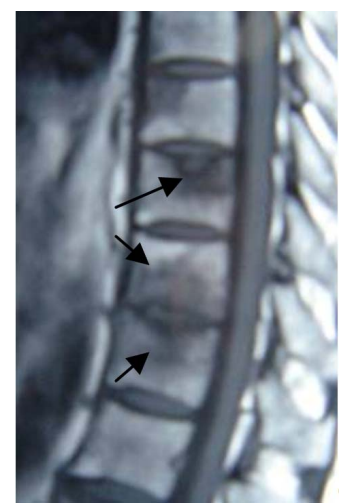

(a)

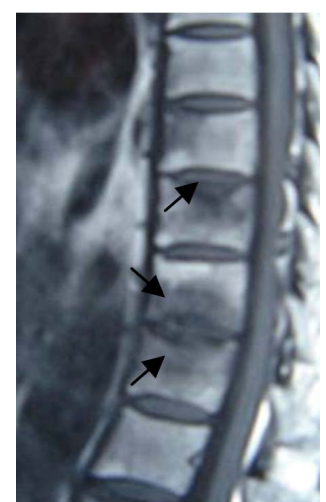

(b)

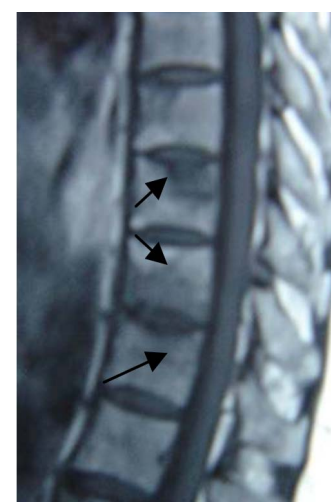

(c)

Figure 5. Patient, female, aged 45, MRI showed L2 and L3, and inflammatory infiltration of disease 4 Brucella spondylitis line drug treatment, after treatment of vertebral and disc space infection improved significantly. (a) Before treatment; (b) After one course of treatment review; (c) Two courses after the review.

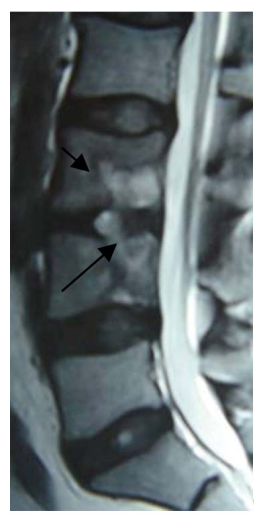

(a)

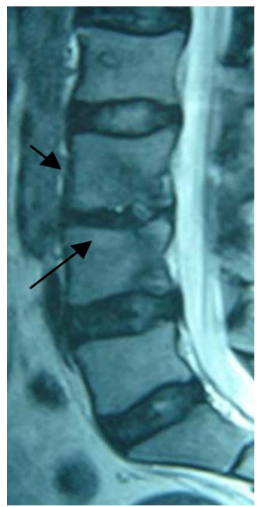

(b)

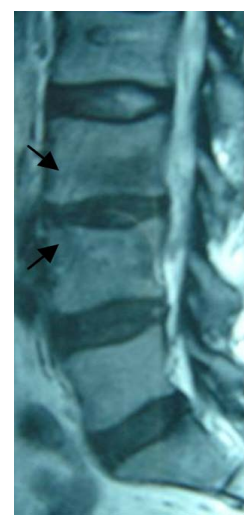

(c)

Figure 6. Patient, female, aged 46, MRI showed the L3, 4 Brucella disease spinal inflammation adjuvant drug therapy compared with before treatment, after treatment vertebral inflammatory infiltration and disc space infection has been absorbed. (a) Pre-treatment; (b) A course of treatment after the review; (c) Two courses after the review. 
continuously [11]. Pay attention to rechecking the hepatic and renal function and finding the preventing the complications in time.

\section{REFERENCES}

[1] M. Turgut, A. T. Turgut and U. Kosar, "Spinal Brucellosis: Turkish Experience Based on 452 Cases Published during the Last Century," Acta Neurochirgica, Vol. 148, No. 10, 2006, pp. 1033-1044. doi:10.1007/s00701-006-0877-3

[2] A. Raptopoulou, A. H. Karantanas, K. Poumboulidis, G. Grollios, M. Raptopoulou-Gigi and A. Garyfallos, "Brucellar Spondylodiscitis: Noncontiguous Multifocal Involvement of the Cervical, Thoracic, and Lumbar Spine," Clinical Imaging, Vol. 30, No. 3, 2006, pp. 214-217. doi:10.1016/j.clinimag.2005.10.006

[3] M. H. Yilmaz, B. Mete, F. Kantarci, R. Ozaras, H. Ozer, A. Mert, I. Mihmanli, R. Ozturk and K. Kanbergoglu, “Tuberculous, Brucellar and Pyogenic Spondylitis: Comparison of Magnetic Resonance Imaging Findings and Assessment of Its Value,” South Medical Journal, Vol. 100, No. 6, 2007, pp. 613-614. doi:10.1097/SMJ.0b013e3180600eaa

[4] J. S. G. del Pozo, M. V. Soto and J. Solera, "Vertebral Osteomyelitis: Long-Term Disability Assessment and Prognostic Factors,” Journal of Infection, Vol. 54, No. 2, 2007, pp. 129-134. doi:10.1016/j.jinf.2006.01.013

[5] P. Atonis, M. Tzermiadianos, A. Gikas, P. Papagelopoulos and A. Hadjipavlou, "Surgical Treatment of Spinal Brucellosis," Clinical Orthopaedics \& Related Research, Vol. 444, 2006, pp. 66-72. doi:10.1097/01.blo.0000203455.59393.9a

[6] J. S. G. del. Pozo, M. V. Soto, M. Lizan-Garcia, E. Martinez-Alfaro, J. C. Segura-Luque and J. Solera-Santos, "Incidence of Infectious Spondylitis in the Province of Albacete (Spain)," Enfermedades Infecciosas y Microbiología Clínica, Vol. 23, No. 9, 2005, pp. 545-550. doi: $10.1157 / 13080265$

[7] E. T. Tali, "Spinal Infections," European Journal of Radiology, Vol. 50, No. 2, 2004, pp. 120-133. doi:10.1016/j.ejrad.2003.10.022

[8] Y. A. Gokhale, A. G. Ambardekar, A. Bhasin, M. Patil, A. Tillu and J. Kamath, "Brucella Spondylitis and Sacroiliitis in the General Population in Mumbai," Journal of the Association of Physicians of India, Vol. 51, 2003, pp. 659-666.

[9] X.-M. Yang, W. Shi and Y.-K. Du, "The Clinical Characteristics and Surgical Treatment of Brucellar Spondylitis," Chinese Journal of Orthopedics, Vol. 28, 2008, pp. 35-40.

[10] X.-M. Yang, W. Shi and Y.-K. Du, "Image Manifestations and Surgical Treatment of Spondylitis Caused by Brucells Infection,” Chinese Journal of Zoonoses, Vol. 23, 2007, pp. 1055-1058.

[11] X.-M. Yang, W. Shi and Y.-K. Du, "Investigation on the Curative of Brucellar Spondylitis,” Chinese Journal of Epidemiology, Vol. 27, 2008, pp. 699-703.
[12] J. M. C. Romero, J. L. R. Salado and F. G. de la Llana, "Differences between Tuberculous Spondylitis and Brucellar Spondylitis,” Anales de Medicina Interna, Vol. 18, No. 6, 2001, pp. 309-311.

[13] D. Ozol, A. Koktener and M. E. Uyar, “Active Pulmonary Tuberculosis with Vertebra and Rib Involvement: Case Report,” South Medical Journal, Vol. 99, No. 2, 2006, pp. 171-173. doi:10.1097/01.smj.0000198642.28149.94

[14] G. Aydin, A. Tosun, I. Keles, E. Ayaslioglu, O. Tosun and S. Orkun, "Brucellar Spondylodiscitis: A Case Report,” International Journal of Clinical Practice, Vol. 60, No. 11, 2006, pp. 1502-1505. doi:10.1111/j.1742-1241.2005.00656.x

[15] K. Z. Yuksel, M. Senoglu, M. Yuksel and M. Gul, "Brucellar Spondylo-Discitis with Rapidly Progressive Spinal Epidural Abscess Presenting with Sciatica,” Spinal Cord, Vol. 44, 2006, pp. 805-808. doi:10.1038/sj.sc.3101938

[16] G. S. Guven, B. Cakir, G. Oz, M. D. Tanriover, E. Turkmen and T. Sozen, "Could Remembering the Prozone Phenomenon Shorten our Diagnostic Journey in Brucellosis?" Rheumatology International, Vol. 26, No. 10, 2006, pp. 933-935. doi:10.1007/s00296-006-0118-3

[17] G. Pappas, S. Seitaridis, N. Akritidis and E. Tsianos, "Treatment of Brucella Spondylitis: Lessons from an Impossible Meta-Analysis and Initial Report of Efficacy of a Fluoroquinolone-Containing Regimen,” International Journal of Antimicrobial Agents, Vol. 24, No. 5, 2004, pp. 502-507. doi:10.1016/j.ijantimicag.2004.05.003

[18] Y. Bayindir, E. Sonmez, A. Aladag and N. Buyukberber, "Comparison of Five Antimicrobial Regimens for the Treatment of Brucellar Spondylitis: A Prospective, Randomized Study," Journal of Chemotherapy, Vol. 15, No. 5, 2003, pp. 466-471.

[19] J. Solera, P. Geijo and J. Largo, “Arandomized, Double-Blind Study to Assess the Optimal Duration of Doxycycline Treatment for Human Brucellosis,” Clinical Infectious Diseases, Vol. 39, No. 12, 2004, pp. 1776-1782. doi:10.1086/426024

[20] E. Alp, R. K. Koc, A. C. Durak, O. Yildiz, B. Aygen, B. Sumerkan and M. Doganay, "Doxycycline plus Streptomycin versus Ciprofloxacin plus Rifampicin in Spinal Brucellosis,” BMC Infectious Diseases, Vol. 6, 2006, pp. 72-78. doi:10.1186/1471-2334-6-72

[21] N. G. David, C. M. Robert, M. E. George and A. S. Merle, "The Sanford Guided to Antimicrobial Therapy," 34th Edition, Antimicrobial Theraphy, Inc., Hyde Park, 2004, pp. 48-51.

[22] Y. Bayindir, E. Sonmez, A. Aladag and N. Buyukberber, "Comparison of Five Antimicrobial Regimens for the Treatment of Brucellar Spondylitis: A Prospective, Randomized Study," Journal of Chemotherapy, Vol. 15, No. 15, 2003, pp. 466-471.

[23] N. Saltoglu, Y. Tasova and A. S. Inal, "Efficacy of Rifampicin plus Doxycycline versus Rifampicin plus Quinolone in the Treatment of Brucellosis," Saudi Medical Journal, Vol. 23, No. 8, 2002, pp. 921-924. 